\title{
México: Valor agregado en las exportaciones manufactureras
}

\author{
Gerardo Fujii G. y Rosario Cervantes $M$.
}

RESUMEN

En las últimas décadas, el sector exportador mexicano ha experimentado un dinamismo y una transformación extraordinarios donde dominan las exportaciones manufactureras, destacándose los productos de tecnología media y alta. Sin embargo, ha crecido la brecha entre exportaciones y producto, lo que indica la debilidad del sector exportador para impulsar el crecimiento económico. El trabajo se basa en la idea de que la capacidad de las exportaciones de dinamizar la economía se refuerza si estas amplían el mercado interno. Ello depende del ingreso nacional contenido en las exportaciones. Se presenta una estimación del valor agregado nacional contenido en las exportaciones manufactureras por sectores y según si es directo -ingreso generado directamente por la actividad exportadora-, o indirecto -ingresos contenidos en los insumos que conforman los productos exportados. La información concierne a las exportaciones manufactureras totales, las de la industria maquiladora de exportación y las del resto de la economía. México.fujii@unam.mx

Rosario Cervantes M. es profesora asociada del Centro Universitario de Ciencias Económico Administrativas de la Universidad de Guadalajara, México.mariac@cucea.udg.mx 


\section{I}

\section{Introducción}

El tema de la relación entre las exportaciones y el crecimiento económico ha sido objeto de muchas investigaciones, tanto teóricas como empíricas. Se han definido cuatro vías fundamentales a través de las cuales se produce esta relación: en primer lugar, la competencia en los mercados internacionales estimula el logro de una mayor eficiencia del aparato productivo (Bhagwati y Srinivasan, 1979; Feder, 1983; Kohli y Singh, 1989; Krueger, 1980). En segundo término, las exportaciones favorecen la especialización, lo que permite beneficiarse de las economías de escala (Helpman y Krugman, 1985). Tercero, las empresas orientadas a la exportación tienden a ser tecnológicamente más avanzadas y su progreso técnico se difunde por el resto de la economía (Grossman y Helpman, 1991). Por último, al dotar de divisas a las economías, las exportaciones hacen posible superar la restricción externa al crecimiento (Thirlwall, 1979). Estos argumentos sirvieron de fundamento para plantear que los países que siguen un modelo de crecimiento liderado por las exportaciones tenderán a crecer más aceleradamente que los que no lo hacen. Por otra parte, en varias investigaciones se ha argumentado que las exportaciones manufactureras son las que más contribuyen al crecimiento en virtud del dinamismo de la demanda mundial por estos productos, del comportamiento de sus precios y debido a las posibilidades de incorporación de progreso técnico derivadas del hecho de contar con un importante sector exportador manufacturero.

Dichos planteamientos encontraron un suelo fértil en muchos países en vista del elevado dinamismo de algunas economías de Asia que, según estas opiniones, se deriva del empuje dado a las exportaciones manufactureras, que habrían sido la fuerza crucial que impulsó el crecimiento de sus economías. Este tema ha adquirido relevancia adicional en el actual contexto de profunda crisis económica que sigue afectando a gran parte del mundo, lo que está llevando a muchos países a intentar una salida por medio del incremento de sus exportaciones.

Estas ideas se difundieron en América Latina a partir de los años ochenta del siglo pasado, lo que condujo a la apertura de las economías de la región y a privilegiar

$\square$ Este trabajo ha sido apoyado por el Consejo Nacional de Ciencia y Tecnología de México a través de los fondos I0017, proyecto 152740. al sector exportador. México ha sido uno de los países que ha mostrado en las últimas décadas un elevado dinamismo exportador y un cambio notable en la composición de las exportaciones en favor de las de origen manufacturero, en particular las de tecnología media y alta. Sin embargo, el crecimiento de largo plazo de la economía mexicana ha sido claramente insuficiente. El objetivo central de este trabajo es contribuir a explicar este comportamiento.

Una de las respuestas a este problema, conectada con el enfoque adoptado en este trabajo, se basa en el hecho de que el crecimiento de las exportaciones ha estimulado la importación de insumos, lo que ha provocado que el efecto multiplicador de las exportaciones en la dinámica de la economía sea bajo (Ruiz Nápoles, 2004; Moreno-Brid, Rivas y Santamaría, 2005; Cervantes, 2008). El presente trabajo tiene como punto de partida la consideración de que el crecimiento de las exportaciones puede generar la expansión del mercado interno. Convencionalmente, el crecimiento liderado por las exportaciones se presenta como opuesto al impulsado por la demanda interna (Eatwell, 1998, págs. 737-738). Esta idea se ha manifestado recientemente en el planteamiento que sostiene que las economías de Asia oriental y China deberían reorientar su crecimiento al mercado interno. Aquí se parte de la idea de que el crecimiento no es necesariamente liderado por las exportaciones, sino que es excluyente con respecto al impulsado por la demanda interna, y que existe una configuración del sector exportador que hace posible que su expansión dinamice al mercado interno (Palley, 2002; Razmi y Blecker, 2008). Por otra parte, entre aquellos que como Felipe (2003, pág. vii), refiriéndose a los países del sudeste de Asia, escriben que "en último término, de lo que se trata es de alcanzar una combinación óptima entre el crecimiento liderado por las exportaciones y el crecimiento liderado por la demanda interna", o como Felipe y Lim (2005, pág. 4) que afirman: "las mejores épocas parecen ser aquellas en que tanto la demanda interna como las exportaciones netas muestran un crecimiento o mejoramiento significativo y persistente", no se destaca con suficiente énfasis, en opinión de los autores de este trabajo, la complementariedad que puede existir entre la expansión de las exportaciones y la dinamización del mercado interno. Esto explica la 
relevancia de calcular el valor agregado, tanto directo como indirecto, contenido en las exportaciones.

Aquí se parte de la idea de que si bien la dinámica de la economía puede estar marcada por el comportamiento de las exportaciones, la expansión de estas no es necesariamente una vía alternativa al crecimiento impulsado por la demanda interna, sino que dicha expansión puede, simultáneamente, contribuir a ampliar tal demanda, lo que permitirá que el crecimiento de la economía sea impulsado tanto por el mercado externo como por el interno. Según se verá en la sección II, este problema ha sido tratado previamente en la literatura desde el punto de vista de la estimación del crecimiento con equilibrio externo. En el presente estudio se enfatiza un aspecto diferente, centrado en el valor agregado nacional, tanto directo como indirecto, contenido en las exportaciones. En la medida en que el ingreso nacional que contienen las exportaciones es más elevado, mayor es la expansión de la demanda interna derivada de las exportaciones. Dado que la concreción de esta posibilidad depende de las características del sector exportador desde la perspectiva del valor agregado contenido en ellas, aquí se hace hincapié en el análisis de las exportaciones manufactureras de México desde dicha perspectiva. En este artículo se plantea que este enfoque podría contribuir, por una parte, a explicar el lento crecimiento experimentado por la economía mexicana en las últimas décadas y, por otra, a precisar algunas características del sector exportador que determinan su transformación en una locomotora para el crecimiento.

En el gráfico 1 se muestran las diversas vías a través de las cuales las exportaciones pueden contribuir al crecimiento económico mediante la dinamización del mercado interno. Por una parte, las exportaciones se traducen de manera directa en empleo, salarios y beneficios (1). El valor agregado directo contenido en las exportaciones genera demanda de bienes de consumo y de capital, la que según la proporción en que es satisfecha mediante producción nacional contribuye a ampliar el mercado interno (2). Por otra, la producción de exportaciones requiere de insumos (3). En la medida en que una proporción más elevada de estos bienes intermedios sea provista por empresas locales (4), mayores serán el empleo, los salarios y los beneficios generados de manera indirecta por el sector exportador (5). En otras palabras, las exportaciones contribuyen a expandir la demanda global y el producto a través de dos vías: por constituir un componente de la demanda global y por el efecto multiplicador que el incremento de las exportaciones tiene en los otros componentes de la demanda agregada. Asimismo, el aumento de las exportaciones requiere de más insumos, los que —en caso de ser producidos dentro del país- elevan la producción de los sectores que los producen y generan un efecto

GRÁFICO 1

\section{Exportaciones y demanda interna}

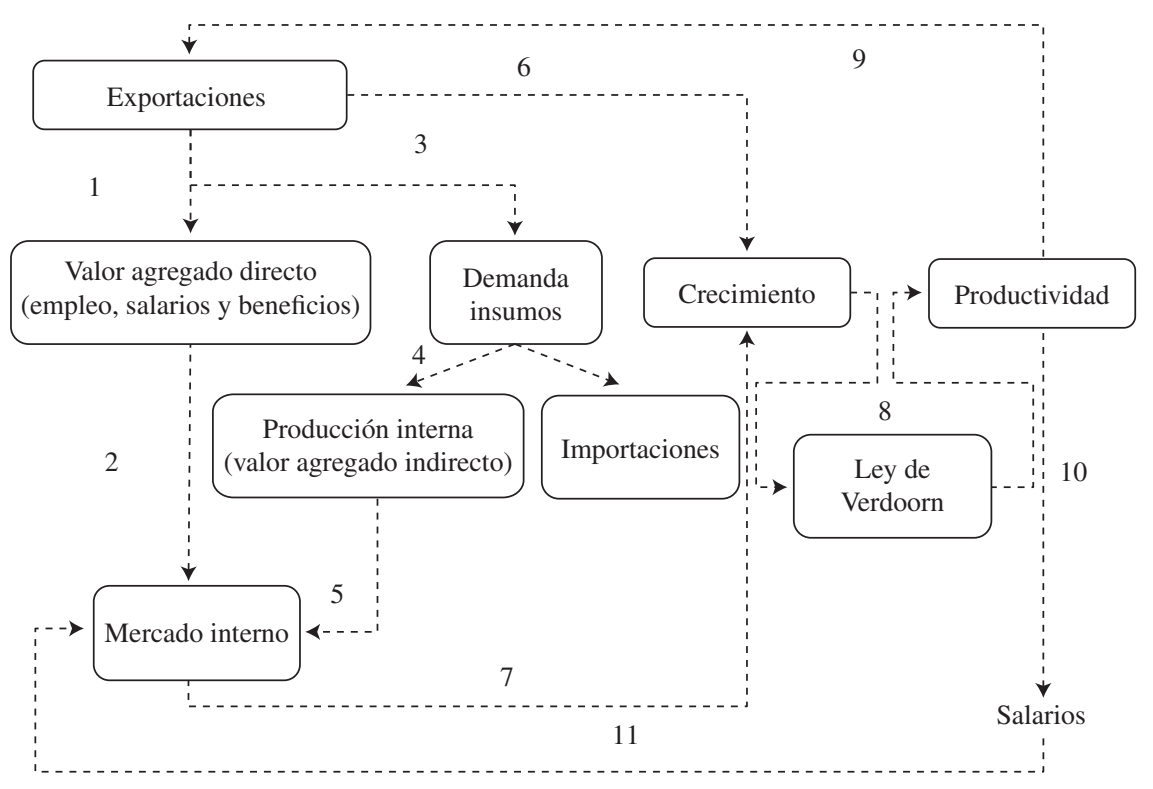

Fuente: elaboración propia. 
multiplicador derivado de su expansión. Entonces, el crecimiento del producto pasa a ser una consecuencia directa de la expansión de las exportaciones (6) y de la ampliación del mercado interno como consecuencia indirecta de las exportaciones a través de los ingresos directos contenidos en ellas y de la demanda por insumos intermedios incorporados en las exportaciones (7). Si además se cumple la Ley de Verdoorn (8), el crecimiento de la productividad permite, por una parte, incrementar la competitividad de los precios y las exportaciones (9) $\mathrm{y}$, por otra, aumentar los salarios (10), lo que impulsa a su vez al mercado interno (11). Con esto, la economía entra en un círculo virtuoso de crecimiento promovido por la demanda.

En el presente trabajo solo se hará referencia a las relaciones del gráfico 1 que están mediadas por el valor agregado contenido en las exportaciones. En este sentido, el objetivo es presentar una estimación del valor agregado de origen nacional contenido en las exportaciones manufactureras de México, ya que su magnitud determina la capacidad que tiene el sector exportador de generar ingresos en forma directa e indirecta $y$, consecuentemente, de expandir el mercado interno y, en último término, de contribuir a crear las condiciones de demanda que estimulen el crecimiento.

Este problema ha adquirido particular relevancia debido a los cambios que se han ido produciendo en la manufactura dentro del sistema de división internacional del trabajo durante las últimas décadas. Sobre todo, la fragmentación del proceso de producción entre fases que se desarrolla en diversos países ha dado lugar a una nueva veta de análisis para cuantificar la contribución de las exportaciones al crecimiento económico. Dado que en muchas economías se ha incrementado el contenido importado de los bienes exportados y que, además, parte de los bienes importados pueden contener productos que antes han sido exportados por la economía que está importando, ha surgido la preocupación por calcular el valor agregado nacional contenido en las exportaciones e importaciones, que son diferentes del valor de estos flujos (Loschky y Ritter, 2006; Breda, Cappariello y Zizza, 2007; Kranendonk y Verbruggen, 2008; Breda y Cappariello, 2008; Koopman, Wang y Wei, 2008; Chen y otros, 2008; Akyüz, 2010). Este problema es especialmente gravitante en los países cuyo sector exportador participa intensamente en el sistema de producción internacional compartida, donde las exportaciones se caracterizan por presentar un elevado componente importado. Uno de estos países es China, lo que recientemente ha dado lugar al desarrollo de enfoques metodológicos y estimaciones del valor agregado nacional contenido en sus exportaciones, segmentando a la economía en dos sectores: uno constituido por las actividades integradas en el sistema de producción internacional compartida y el otro que comprende el resto de la economía (Chen y otros, 2005; Koopman, Wang y Wei, 2008; Daudin, Rifflart y Schweisguth, 2009; He y Zhang, 2010).

México es uno de los países que se han incorporado al sistema de producción internacional compartida, lo que hace que sea muy relevante estimar cuál es el valor agregado nacional contenido en sus exportaciones manufactureras. Esto puede contribuir a estimar con mayor precisión el aporte real de las exportaciones al crecimiento económico del país por parte de la expansión de la demanda interna derivada de las exportaciones.

La estructura de este artículo es la siguiente: en la sección II se expone brevemente la línea de razonamiento seguida en la literatura sobre la relación entre exportaciones y crecimiento económico, desde el punto de vista de las vías a través de las cuales las exportaciones pueden contribuir a la expansión de la demanda interna y, por lo tanto, al crecimiento de la economía. Además, se señala la nueva perspectiva con que se está abordando el tema en el contexto de la intensificación de la producción internacional compartida. En la sección III se describe el método con que se estimó el valor agregado nacional contenido en las exportaciones manufactureras de México. En la sección IV se presentan, en forma sintética, las transformaciones que ha experimentado el sector exportador mexicano y la notable divergencia verificada en las últimas décadas entre la dinámica de las exportaciones y la de la producción. En la sección $\mathrm{V}$ se presenta la estimación del valor agregado nacional contenido en las exportaciones, elemento que determina la magnitud en que las exportaciones dinamizan al mercado interno y, por lo tanto, al conjunto de la economía. El trabajo finaliza con la presentación de sus conclusiones. 


\section{II}

\section{Exportaciones, demanda interna y crecimiento}

Ya Adam Smith se refirió a que el comercio exterior, al ampliar el mercado, contribuye a incrementar la producción por la vía de las exportaciones, lo que a su vez permite profundizar la división del trabajo, factor que - en su planteamiento — es clave para aumentar la riqueza de las naciones. En palabras de Smith, a través del comercio exterior los países ".... remiten al exterior el excedente del producto de su tierra y de su trabajo, carente de demanda en el interior, y consiguen traer, a cambio de aquel excedente, artículos que se solicitan en el país... Gracias al comercio exterior, la limitación del mercado doméstico no impide que la división del trabajo, en una rama particular de las artes y las manufacturas, sea llevada hasta su máxima perfección. Al abrir un mercado más amplio para cualquier porción del producto del trabajo que exceda las necesidades del consumo doméstico, el comercio exterior sirve de estímulo para perfeccionar y fomentar las fuerzas productivas de manera que el producto anual alcance un desarrollo considerable y, por consiguiente, la riqueza y la renta efectiva de la sociedad" (Smith, 1958).

La vía convencional a través de la cual la economía ha abordado el tema de la relación entre exportaciones y crecimiento desde la perspectiva de la demanda, ha sido mediante el efecto de las exportaciones en los componentes de la demanda global, tanto en forma directa, porque las exportaciones son un componente de la demanda global, como indirecta, por el efecto multiplicador que las exportaciones tienen en otros componentes de la demanda global. La vía convencional a través de la cual en la economía se ha abordado el tema de la relación entre exportaciones y crecimiento desde la perspectiva de la demanda, ha sido por el efecto de las exportaciones en los componentes de la demanda global, tanto en forma directa, porque las exportaciones son un componente de la demanda global, como indirecta, por el efecto multiplicador que las exportaciones tienen en otros componentes de la demanda global. Este enfoque está presente en los conceptos de multiplicador del comercio exterior (Harrod, 1933). Y también lo está en el concepto del super-multiplicador del comercio exterior (Hicks, 1950), que al efecto que las exportaciones ejercen en el producto - a través del multiplicador del comercio exterior de Harrod- suma el hecho de que el incremento de las exportaciones permite que se expandan otros componentes de la demanda, hasta el punto en que el aumento de las importaciones sea igual al acrecentamiento inicial de las exportaciones. En esta misma perspectiva están los trabajos de Thirlwall (1979) y de Kaldor (1989), cuando estiman el crecimiento del producto que genera una tasa dada de expansión de las exportaciones, la que está mediada por el incremento de las importaciones. En términos de Kaldor "desde el punto de vista de cualquier región en particular, el 'componente autónomo de la demanda' es la demanda que emana desde fuera de la región; y el concepto del 'super-multiplicador' de Hicks puede ser aplicado para expresar la idea del multiplicador del comercio exterior en un contexto dinámico. Así expuesta, esta noción afirma que la tasa de desarrollo económico de una región está, en lo fundamental, determinada por la tasa de crecimiento de sus exportaciones" (Kaldor, 1989, pág. 318). Aún más, el autor extiende el mismo principio a los países en desarrollo: "Para ser exitosa, la difusión de la industrialización entre los países en desarrollo implica seguir una 'estrategia orientada hacia afuera' que conduzca al desarrollo del potencial exportador y no limitarse solamente a la sustitución de importaciones..." (Kaldor, 1989, pág. 341).

De acuerdo con las ideas expuestas, en la estimación de la contribución al crecimiento de las exportaciones no se considera la nueva faceta de la división internacional del trabajo por fases del proceso productivo de un mismo producto, lo que determina la relevancia de calcular el valor agregado nacional contenido en los flujos de comercio. 


\section{III \\ Método de cálculo del valor agregado nacional contenido en las exportaciones}

El análisis insumo-producto permite calcular el valor agregado nacional contenido en las exportaciones manufactureras; este puede ser dividido en dos partes: el valor agregado directo, equivalente a los ingresos generados durante el proceso de transformación de insumos en productos terminados para la exportación; y el valor agregado indirecto, que corresponde a los ingresos que se generaron durante la producción de esos insumos de origen nacional que se incorporan a las exportaciones.

Con la matriz insumo-producto para México, elaborada por el Instituto Nacional de Estadística y Geografía (INEGI) con datos de 2003 (INEGI, 2008), se pueden hacer estas estimaciones en forma separada para la industria maquiladora de exportación (IME) y para el resto de las actividades exportadoras incluidas en lo que el INEGI denomina economía interna (EI).

Para hacer la estimación se seguirá la metodología empleada por Koopman, Wang y Wei (2008) y He y Zhang (2010) para el cálculo del valor agregado nacional contenido en las exportaciones manufactureras de China. En estos trabajos las exportaciones son divididas entre "exportaciones ordinarias" y "exportaciones en proceso", las que se diferencian básicamente por la proporción de insumos importados que se incorporan en la producción. Estas expresiones son equivalentes a las de "exportaciones de la economía interna" y "de la industria maquiladora de exportación" empleadas en la matriz de México.

En la metodología se procura estimar el efecto de las exportaciones de un sector cualquiera en el valor agregado de otros sectores a través de la demanda de insumos intermedios.

El valor agregado nacional contenido en las exportaciones se descompone en dos partes: una fracción generada por las exportaciones de la EI y, la otra, por las exportaciones de la IME.

El valor agregado directo e indirecto contenido en las exportaciones de la EI se estima a partir de los multiplicadores de valor agregado representados en la ecuación (1); mientras que el valor agregado directo e indirecto generado por las exportaciones de la IME se estima a partir de la ecuación (2).

$$
\begin{gathered}
M^{E I}=A_{V}^{E I}\left(I-A^{E I}\right)^{-1} \\
M^{I M E}=\left[A_{V}^{E I}\left(I-A^{E I}\right)^{-1} A^{I M E}+A_{V}^{I M E}\right]
\end{gathered}
$$

En (1), $M^{E I}$ es la matriz de coeficientes de valor agregado contenido en las exportaciones de las empresas de la EI. Mientras que en (2), $M^{I M E}$ es la matriz de coeficientes de valor agregado de las exportaciones de la IME. Ambas matrices son de dimensión $r \mathrm{x} r$, donde $r$ representa el total de subsectores de la economía.

$A_{V}^{E I}$ es una matriz diagonal de coeficientes de valor agregado de la EI, cuyos elementos en la diagonal principal se obtienen al dividir el valor agregado total del subsector por el valor bruto de la producción del mismo subsector; $\left(I-A^{E I}\right)^{-1}$ es la matriz inversa de Leontief que, en el caso de la economía mexicana, se obtiene a partir de los coeficientes de insumos de la EI, esto es, descontando el consumo intermedio de la IME, pues las empresas de este sector no participan en la producción de insumos intermedios, solo los utilizan.

En la ecuación (2), $A_{V}^{I M E}$ es una matriz diagonal de coeficientes de valor agregado directo generado por las actividades de la IME, que resulta de dividir el valor agregado total de cada subsector por el valor bruto de la producción; en el caso de las empresas de la IME, es igual al volumen de sus exportaciones. $A^{I M E}$ es una matriz de coeficientes de insumos intermedios de origen nacional que demanda la IME.

$\mathrm{Al}$ sumar por columnas los valores que se obtienen en la matriz $M^{E I}$ se derivan los multiplicadores de valor agregado de las exportaciones de las empresas de la EI para cada subsector. Mientras que las sumas por columna de la matriz $M^{I M E}$ representan los multiplicadores de valor agregado de las exportaciones de la IME. 


\section{IV}

\section{México: dinamismo y cambio en la composición de las exportaciones con lento crecimiento}

A partir del último tramo de los años ochenta del siglo pasado, en México se ha intentado seguir una estrategia de crecimiento liderada por las exportaciones manufactureras. Se verá que efectivamente estas se han acrecentado a una enorme velocidad, pero no han logrado convertirse en el motor del crecimiento del país.

\section{Exportaciones totales}

El comienzo del proceso de liberalización comercial de México se puede fechar en 1987, año en que el país adhirió al Acuerdo General sobre Aranceles Aduaneros y Comercio (GATT). Entre 1992 y 2008, el total de exportaciones del país se expandió en forma notable, pasando de 46.200 a 291.000 millones de dólares en un período de 16 años. La tasa media anual de incremento de las exportaciones en el lapso 1989-2006 fue de 9,6\%; entre 1989 y 1993 , de $5,8 \%$ y de 1994 a 2008 , de $14,1 \%$. Esto condujo a un notable incremento del coeficiente de exportaciones del país, que pasó de un $13 \%$ a alrededor del 27\% entre los años 1992 y 2008 (véase el gráfico 2).

\section{Cambio en la composición de las exportaciones}

El dinamismo exportador del país fue simultáneo a la modificación en la composición de las exportaciones de bienes, de manera que en 2008 las exportaciones de origen manufacturero ascendían a 231.000 millones de dólares, equivalentes al $79 \%$ del total de las exportaciones del país (véase el gráfico 3).

\section{Exportaciones manufactureras por intensidad de uso de factores}

En el gráfico 4 se observa la composición de las exportaciones industriales por intensidad de uso de factores, clasificadas entre productos intensivos en recursos naturales o en tecnología, ya sea baja, media o alta. Se constata que el peso decisivo le corresponde a los productos industriales intensivos en tecnología media y alta, que ya desde comienzos de la década de 1990 han representado alrededor del $60 \%$ de las exportaciones industriales del país. Sin embargo, estos datos deben ser considerados

GRÁFICO 2

México: exportaciones totales y coeficiente de exportación

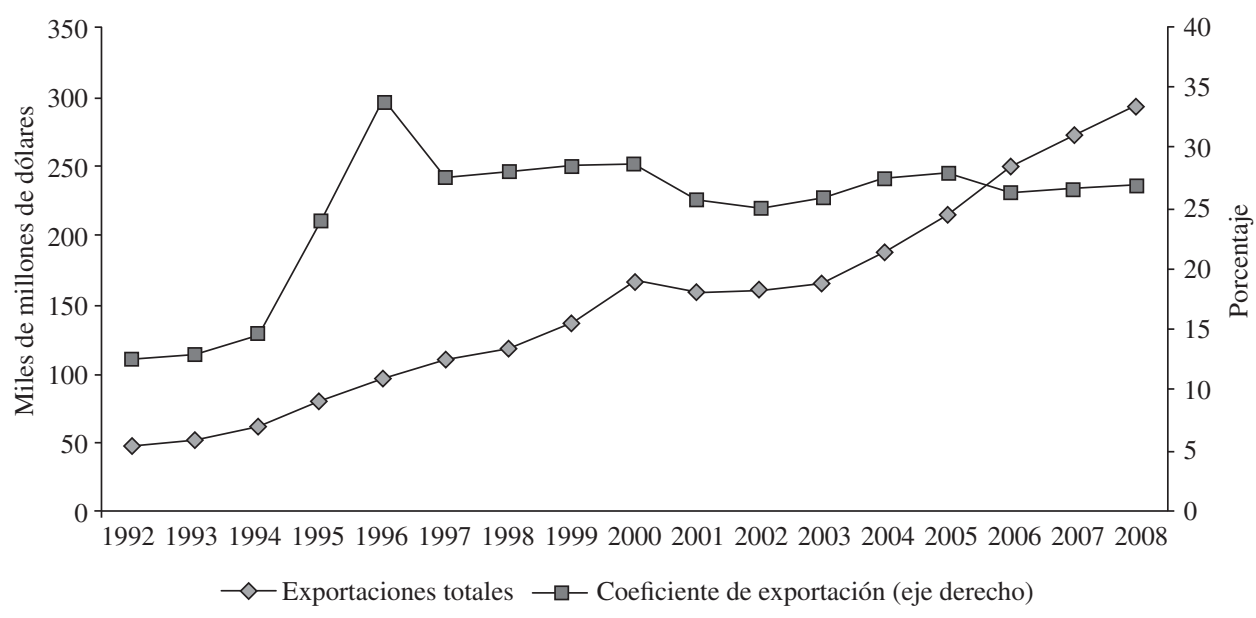

Fuente: Banco de México, Informe anual, 2008, México, D.F., 2009. 
GRÁFICO 3

\section{México: exportaciones por tipos de mercancías, 2008 \\ (En porcentajes)}

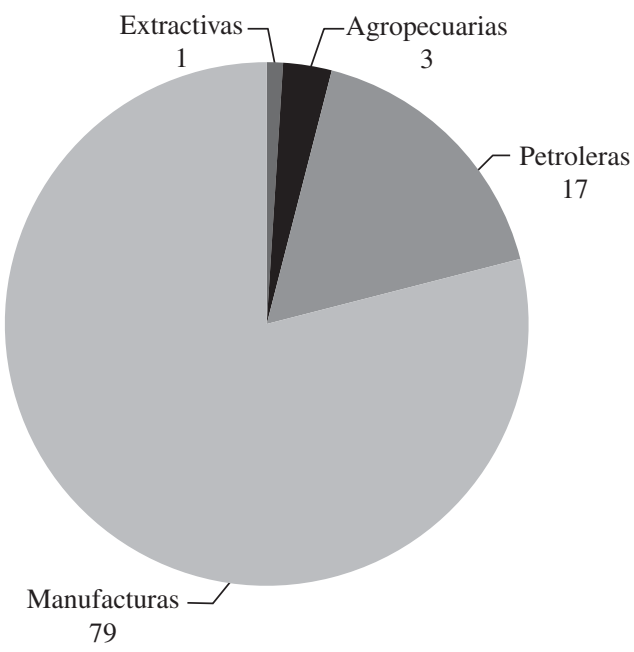

Fuente: Banco de México, Informe anual, 2008, México, D.F., 2009.

con cuidado, pues se derivan de la clasificación de los productos exportados por nivel tecnológico y bien puede ocurrir que un país esté especializado en la fase de producción tecnológicamente simple de un producto que es de elevada tecnología. Esto es sobre todo importante de considerar en países en que parte importante de las exportaciones manufactureras son generadas bajo el esquema de la producción internacional compartida, en el que, como se verá enseguida, México participa intensamente.

El no considerar la particularidad del perfil exportador manufacturero de países muy integrados al sistema de producción internacional compartida puede dar lugar a equívocos. Por ejemplo, Myro y otros (2008, págs. 38 y 40) clasifican las exportaciones manufactureras de los países integrantes de la Organización de Cooperación y Desarrollo Económicos (OCDE) en tres grupos - avanzadas, intermedias y tradicionales-, y según el dinamismo de la demanda y la intensidad tecnológica, destacan que en 2005 el $41 \%$ de las exportaciones manufactureras de México se encontraban en el primer grupo, el $39 \%$ fueron de tipo intermedio y el $25 \%$ se hallaban en la categoría de exportaciones tradicionales. Los mismos datos para Alemania son $21 \%, 55 \%$ y $23 \%$, respectivamente, mientras que para el Japón los datos correspondientes ascienden a $32 \%, 55 \%$ y $13 \%$, respectivamente. En suma, estos antecedentes mostrarían que la inserción internacional
GRÁFICO 4

México: exportaciones industriales por contenido tecnológico, 2006

(En porcentajes de las exportaciones industriales totales)

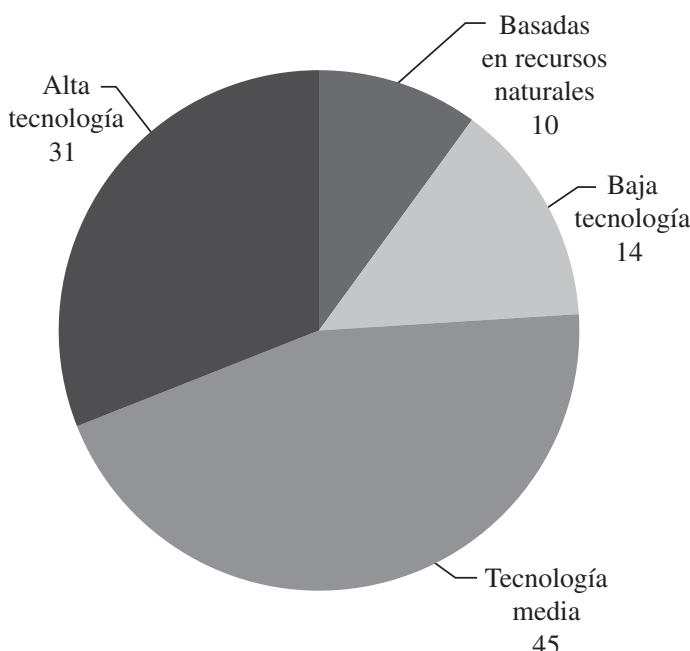

Fuente: Comisión Económica para América Latina y el Caribe (CEPAL), Panorama de la inserción internacional de América Latina y el Caribe 2007. Tendencias 2008 (LC/G.2383-P), Santiago de Chile, 2008. Publicación de las Naciones Unidas, $\mathrm{N}^{\mathrm{o}}$ de venta: S.08.II.G.36.

de México a través de las exportaciones manufactureras es más avanzada —en términos tecnológicos y debido al dinamismo de la demanda - que las de dos grandes potencias exportadoras desarrolladas. Y no solo ello, sino que además, de acuerdo con la sofisticación de sus exportaciones (Hausmann, Hwang y Rodrik, 2007) y el índice de adaptabilidad que muestra el país, su inserción internacional debiera crecer más ya que cuenta con la especialización productiva adecuada. En estas afirmaciones no se toma en consideración la fase del proceso de producción de los productos de alta tecnología en las que México se ha especializado.

No obstante el comportamiento extraordinario que ha mostrado el sector manufacturero exportador mexicano a partir de los años ochenta del siglo pasado, la brecha entre exportaciones y producto no ha cesado de ampliarse en las últimas décadas (véase el gráfico 5). Este hecho, destacado por Palma (2005), fue particularmente notable en el decenio de 1990, en que las exportaciones aumentaron a una tasa media anual de $12,5 \%$, mientras que el producto lo hacía a un ritmo de 3,4\% (Banco Mundial, 2011). 


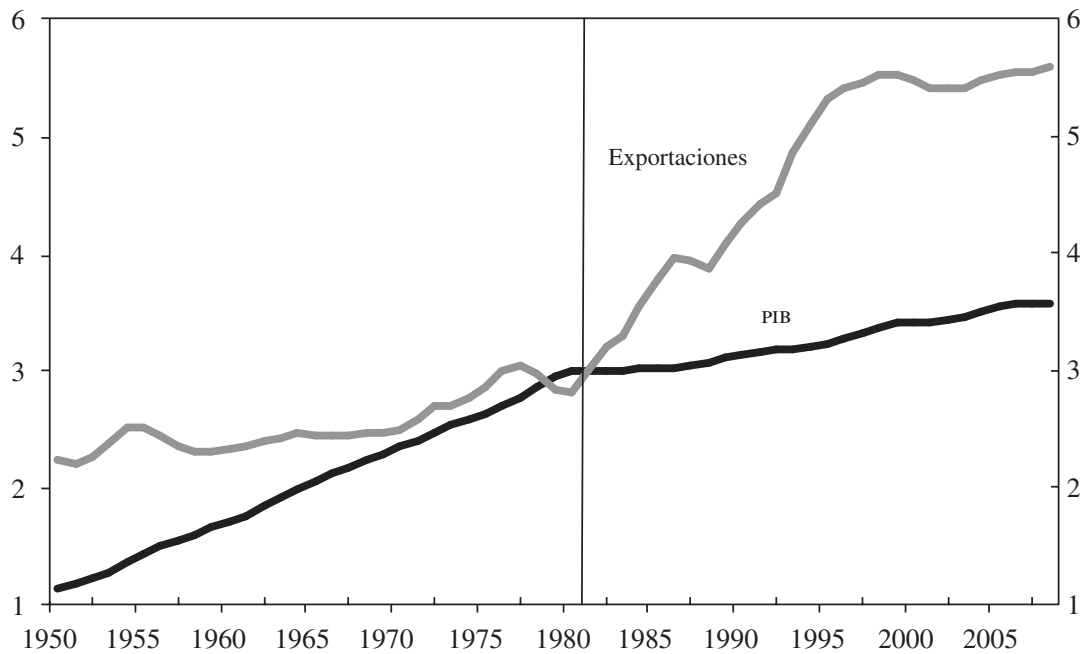

Fuente: Instituto Nacional de Estadística y Geografía (INEGI), "Banco de información económica" para las series 1980-2010; "Estadísticas históricas de México", Petróleos Mexicanos para la serie 1950-1979 del PIB; y Pemex (1988), Anuario estadístico, 1988, para la serie de las exportaciones petroleras 1950-1979.

a Las cifras presentadas están calculadas como promedios móviles para períodos de 3 años, que luego son transformados en un número índice en base logarítmica. Se toma como base el año $1981=20,1$, tanto para el valor observado del PIB, como para el valor observado de las exportaciones no petroleras.

\section{V}

\section{Estimación del valor agregado nacional contenido en las exportaciones manufactureras de México}

En virtud de que las características de las exportaciones manufactureras generadas por la EI y la IME determinan que el valor agregado contenido en ellas sea muy diferente, tanto en magnitud como en la proporción en que se genera en forma directa e indirecta, se presenta en primer lugar una descripción somera del peso que ambos sectores tienen en las exportaciones manufactureras del país, tanto en el conjunto como a nivel de las ramas de la manufactura.

\section{Exportaciones manufactureras de la economía interna (EI) y de la industria maquiladora de exportación (IME)}

En el cuadro 1 se muestra la composición de las exportaciones manufactureras del país por subsectores de actividad económica y se detallan los tres sectores que contribuyen con dos tercios de las exportaciones mexicanas: industria de computación y electrónica, industria de equipos de transporte e industria eléctrica, y según si provienen de la IME o de la EI (en el cuadro A1 del anexo estadístico se detalla esta información para 21 sectores; en lo subsecuente, en los cuadros 1 y 2 se muestra la información reducida a los tres sectores más importantes en términos de exportaciones. Los detalles para todos los sectores manufactureros se muestran en los cuadros del anexo estadístico). Los datos más destacados son los siguientes:

- La mayor parte de las exportaciones manufactureras provienen de la industria maquiladora (62\% del total).

- Si los sectores exportadores son estratificados según su nivel de integración con la economía por la contribución de las exportaciones de la EI en tres niveles —alto (más del 70\% de las exportaciones 
provienen de la EI); medio (entre $30 \%$ y $70 \%$ provienen de la EI); y bajo-, se observa que el $52 \%$ de las exportaciones manufactureras del país se originan en sectores con bajo nivel de integración interna. En el otro extremo, solo el 10\% de ellas provienen de sectores altamente integrados con la EI.

- Son tres sectores los que aportan la mayor parte de las exportaciones manufactureras: la industria electrónica (29\% del total), la que produce equipos de transporte (28\%) y la de equipos eléctricos (9\% del total). En conjunto, contribuyen con el $66 \%$ del valor de las exportaciones manufactureras del país.

- Sin embargo, estos sectores son radicalmente diferentes debido a su grado de integración con el resto de la economía nacional: mientras que el $88 \%$ de las exportaciones de la industria electrónica y el $81 \%$ de las de la industria eléctrica son generadas por la industria maquiladora, en el caso de equipos de transporte el $58 \%$ corresponde a la EI.

\section{Valor agregado nacional en las exportaciones manufactureras}

En los cuadros 2 y A2 del anexo se muestra el valor agregado nacional contenido en las exportaciones manufactureras. La información se presenta para el conjunto de la economía y a continuación se descompone entre EI e IME y por sectores de la industria manufacturera. En segundo lugar, se distingue entre valor agregado nacional directo e indirecto. Por último, se presentan los coeficientes de valor agregado nacional contenido en las exportaciones. Con el fin de calificar los datos de la economía mexicana, en los casos en que se dispuso de datos comparables, se exponen los antecedentes correspondientes a la economía china.
Las conclusiones más relevantes que se desprenden de estos cuadros son las siguientes:

i. Las exportaciones de la EI, que constituyen el $38 \%$ de las exportaciones manufactureras, aportan el $67 \%$ del valor agregado interno contenido en las exportaciones manufactureras. Por su parte, la IME, que contribuye con el $62 \%$ de las exportaciones manufactureras, aporta el $33 \%$ del valor agregado nacional contenido en ellas. En otras palabras, el peso de las exportaciones manufactureras radica en el sector que hace una contribución relativamente reducida al ingreso nacional.

ii. Los tres sectores decisivos en materia de exportaciones manufactureras (66\% del total) aportan el 54\% del valor agregado nacional contenido en las exportaciones. O sea, nuevamente se aprecia la debilidad relativa de los sectores decisivos en materia de exportaciones en términos de su aporte al ingreso nacional.

iii. La contribución de cada uno de estos sectores en términos de exportaciones y de valor agregado doméstico es marcadamente diferente: mientras que la producción de equipos de transporte representa el 28\% de las exportaciones manufactureras, su aporte al valor agregado interno contenido en ellas es del 32\%; en la producción de equipos de computación y electrónicos estas variables están en la relación opuesta: este sector genera el $29 \%$ de las exportaciones manufactureras y aporta el $14 \%$ del valor agregado nacional contenido en el total de exportaciones de la manufactura.

iv. Existe una amplia diferencia entre la EI y la IME en cuanto a la participación de estos tres sectores en las exportaciones y en el valor agregado nacional contenido en ellas. En la EI estos sectores aportan el 52\% del valor agregado, mientras que representan el $56 \%$ de las exportaciones, en tanto que en la IME contribuyen con

México: composición de las exportaciones de la industria manufacturera, 2003 (En millones de pesos)

\begin{tabular}{|c|c|c|c|c|c|c|c|c|}
\hline \multirow[b]{2}{*}{ Subsector } & \multicolumn{2}{|c|}{ Total } & \multicolumn{2}{|c|}{$\begin{array}{c}\text { Economía interna } \\
\text { (EI) }\end{array}$} & \multicolumn{2}{|c|}{$\begin{array}{l}\text { Industria maquiladora } \\
\text { de exportación (IME) }\end{array}$} & \multicolumn{2}{|c|}{ Porcentajes } \\
\hline & Exportaciones & Porcentaje & Exportaciones & Porcentaje & Exportaciones & Porcentaje & EI & IME \\
\hline Fabricación de equipos electrónicos & 385317 & 28,9 & 47741 & 9,4 & 337576 & 40,8 & 12,4 & 87,6 \\
\hline Fabricación de equipos de transporte & 366969 & 27,5 & 211203 & 41,6 & 155766 & 18,8 & 57,6 & 42,4 \\
\hline Fabricación de equipos eléctricos & 122366 & 9,2 & 23135 & 4,6 & 99231 & 12 & 18,9 & 81,1 \\
\hline Subtotal tres sectores & 874651 & 65,5 & 282078 & 55,6 & 592573 & 71,6 & 32,3 & 67,7 \\
\hline Resto de industrias manufactureras & 460514 & 34,5 & 225015 & 44,4 & 235499 & 28,4 & 48,9 & 51,1 \\
\hline Total exportaciones & 1335165 & 100 & 507093 & 100 & 828072 & 100 & 38 & 62 \\
\hline
\end{tabular}

Fuente: estimación de los autores en base en Instituto de Estadística y Geografía (INEGI), "Matriz de insumo-producto 2003”, México, D.F., 2008. 
México: valor agregado nacional total contenido en las exportaciones manufactureras, 2003 (En millones de pesos)

\begin{tabular}{|c|c|c|c|c|c|c|c|c|c|c|c|}
\hline & \multicolumn{11}{|c|}{ Total industria manufacturera } \\
\hline & \multicolumn{2}{|c|}{$\begin{array}{l}\text { Valor agregado } \\
\text { total }\end{array}$} & \multicolumn{2}{|c|}{$\begin{array}{l}\text { Valor agregado } \\
\text { directo }\end{array}$} & \multicolumn{2}{|c|}{$\begin{array}{l}\text { Valor agregado } \\
\text { indirecto }\end{array}$} & \multicolumn{5}{|c|}{$\begin{array}{l}\text { Porcentaje de valor agregado nacional } \\
\text { sobre el valor de las exportaciones }\end{array}$} \\
\hline & Pesos & Porcentaje & Pesos & Porcentaje & Pesos & Porcentaje & Total & Directo & Indirecto & $\begin{array}{c}\text { Indirecto } \\
\text { manufac- } \\
\text { turero }\end{array}$ & $\begin{array}{c}\text { Indirecto } \\
\text { intra- } \\
\text { industrial }\end{array}$ \\
\hline Fabricación de equipos de transporte & 182741 & 32,4 & 100446 & 33,4 & 82294 & 31,1 & 49,8 & 27,4 & 22,4 & 5,7 & 2,7 \\
\hline Fabricación de equipos electrónicos & 81024 & 14,3 & 48505 & 16,1 & 32520 & 12,3 & 21 & 12,6 & 8,4 & 1,8 & 0,5 \\
\hline Fabricación de equipos eléctricos & 41578 & 7,4 & 23002 & 7,7 & 18576 & 7 & 34 & 18,8 & 15,2 & 3,8 & 0,2 \\
\hline Subtotal tres sectores & 305343 & 54,1 & 171953 & 57,2 & 133390 & 50,5 & 34,9 & 19,7 & 15,3 & 3,7 & 1,5 \\
\hline Resto de industrias manufactureras & 259416 & 45,9 & 128596 & 42,8 & 130820 & 49,5 & 56,3 & 27,9 & 28,4 & 5,5 & 5,3 \\
\hline Total valor agregado & 564759 & 100 & 300549 & 100 & 264210 & 100 & 42,3 & 22,5 & 19,8 & 4,3 & \\
\hline Fabricación de equipos de transporte & 144396 & 38,1 & 74718 & 39,4 & 69678 & 36,8 & 68,4 & 35,4 & 33 & 8,9 & 4,6 \\
\hline Fabricación de equipos electrónicos & 33812 & 8,9 & 20878 & 11 & 12934 & 6,8 & 70,8 & 43,7 & 27,1 & 7 & 2,8 \\
\hline Fabricación de equipos eléctricos & 17551 & 4,6 & 9398 & 5 & 8153 & 4,3 & 75,9 & 40,6 & 35,2 & 10,4 & 0,5 \\
\hline Subtotal tres sectores & 195759 & 51,7 & 104993 & 55,4 & 90766 & 47,9 & 69,4 & 37,2 & 32,2 & 8,7 & 4,2 \\
\hline Resto de industrias manufactureras & 183185 & 48,3 & 84452 & 44,6 & 98734 & 52,1 & 81,4 & 37,5 & 43,9 & 8,5 & 8,2 \\
\hline \multirow[t]{2}{*}{ Total valor agregado } & 378945 & 100 & 189445 & 100 & 189499 & 100 & 74,7 & 37,4 & 37,4 & 8,6 & \\
\hline & \multicolumn{11}{|c|}{ Industria maquiladora de exportación (IME) } \\
\hline Fabricación de equipos de transporte & 38344 & 20,6 & 25728 & 23,2 & 12616 & 16,9 & 24,6 & 16,5 & 8,1 & 1,2 & 0,1 \\
\hline Fabricación de equipos electrónicos & 47212 & 25,4 & 27627 & 24,9 & 19585 & 26,2 & 14 & 8,2 & 5,8 & 1,1 & 0,2 \\
\hline Fabricación de equipo eléctrico & 24027 & 12,9 & 13604 & 12,2 & 10423 & 14 & 24,2 & 13,7 & 10,5 & 2,2 & 0,1 \\
\hline Subtotal tres sectores & 109584 & 59 & 66960 & 60,3 & 42624 & 57,1 & 18,5 & 11,3 & 7,2 & 1,3 & 0,2 \\
\hline Total valor agregado & 185815 & 100 & 111104 & 100 & 74711 & 100 & 22,4 & 13,4 & 9 & 1,6 & \\
\hline
\end{tabular}

Fuente: estimación de los autores sobre la base de Instituto de Estadística y Geografía (INEGI), "Matriz de insumo-producto 2003", México, D.F., 2008.

el $72 \%$ de las exportaciones, en las que está contenido el $59 \%$ del valor agregado nacional contenido en las exportaciones de la industria maquiladora.

v. En la EI, la mayor diferencia en estos indicadores se presenta en el sector de equipos de transporte (42\% de las exportaciones y 38\% del valor agregado nacional), mientras que en la IME el mayor contraste se da en la electrónica, que contribuye con el $41 \%$ de las exportaciones de la industria maquiladora y aporta el $25 \%$ al valor agregado nacional generado por este sector de la economía.

vi. Los restantes sectores manufactureros hacen contribuciones relativamente pequeñas, tanto en términos de exportaciones como de valor agregado nacional contenido en ellas. En general, el aporte de dichos sectores en términos de valor agregado supera al que hacen en cuanto a exportaciones. Estas brechas son particularmente amplias en los sectores manufactureros que procesan directamente recursos naturales: es el caso de la industria alimentaria $(3,3 \%$ comparado con $1,8 \%$ en valor agregado y exportaciones, respectivamente); de la química $(6,4 \%$ en comparación con $3,5 \%)$; y de la metálica básica $(4,6 \%$ respecto de $2,6 \%)$.

vii. El valor agregado nacional contenido en el total de exportaciones del país asciende al 55\% (cálculo de los autores), sensiblemente superior al coeficiente que corresponde a las exportaciones manufactureras, dado que las de productos agrícolas, mineras y de petróleo tienen coeficientes de valor agregado con respecto al valor de las exportaciones más elevados que los de la manufactura. Respecto de China, esta proporción 
asciende al 47\% (Chen y otros, 2008, pág.14), diferencia que en parte importante debe explicarse por el hecho de que las exportaciones de productos basados en recursos naturales tienen en México un mayor peso en las exportaciones que las de la economía china.

viii. En el sector manufacturero, el valor agregado de origen nacional representa el $42 \%$ del valor de las exportaciones de manufacturas. Esta proporción es significativamente mayor en las exportaciones de la EI $(75 \%)$ que en la IME (22\%).

ix. En China, en el año 2002, los coeficientes de valor agregado nacional como proporción de las exportaciones manufactureras ascendían al $51 \%$ respecto del total de las exportaciones manufactureras; al $88 \%$ con relación a las exportaciones de la ei y al $25 \%$ con respecto al sector procesador de exportaciones (Koopman, Wang y Wei, 2008, pág. 24). Es decir, las proporciones de valor agregado nacional contenido en las exportaciones manufactureras chinas son más elevadas que en México, sobre todo respecto del total de las exportaciones y en relación con las de la EI.

$\mathrm{x}$. En los tres sectores que hacen las mayores contribuciones a las exportaciones manufactureras de México, este coeficiente asciende al $50 \%$ en la fabricación de equipos de transporte, al $21 \%$ en la industria electrónica, y al 34\% en equipos eléctricos. En todos los casos, el coeficiente de valor agregado nacional contenido en las exportaciones de la EI es sustancialmente más alto que en la IME. Respecto de la producción de equipos de transporte esta relación es del $68 \%$ en la EI y de $25 \%$ en la IME. En la industria electrónica estas proporciones ascienden al $71 \%$ (EI) y al 14\% (IME). Por último, en la industria productora de equipos eléctricos la relación es del $76 \%$ (EI) y del $24 \%$ (IME). Los porcentajes correspondientes a estos tres sectores en la industria procesadora de exportaciones de la economía china ascendían al $27 \%, 20 \%$ y $26 \%$, respectivamente (Chen y otros, 2008, pág. 14).

xi. Como se ha expuesto, el valor agregado generado por un sector puede dividirse entre directo, correspondiente a los ingresos factoriales pagados directamente por el sector, e indirecto, que equivale a los ingresos contenidos en los insumos que demanda el sector de que se trate. A su vez, el valor agregado indirecto puede ser nacional, cuando estos insumos tengan su origen dentro del país, o constituir ingresos para otros países si los insumos son importados. Para el caso de las actividades exportadoras, si ellas están intensamente conectadas con el resto de la economía en cuanto a las compras de insumos, se multiplica el efecto generador de ingresos internos derivados de la exportación. Con respecto a las exportaciones manufactureras de México, el 53\% del valor agregado nacional contenido en ellas es directo. Esta proporción es menos elevada en las exportaciones generadas por la EI (50\%) que en las de la IME (60\%).

xii. La proporción de valor agregado nacional indirecto con respecto al valor de las exportaciones manufactureras es, en México, del 20\%, mientras que en China asciende al 32\% (véase el párrafo anterior ix, y también Koopman, Wang y Wei, 2008, pág. 24; datos para 2002 de valor agregado nacional total y valor agregado directo), de lo que se deriva que sus exportaciones tienen una mayor capacidad para generar ingresos indirectos en otros sectores de la economía.

xiii. En los tres sectores decisivos en materia de exportaciones manufactureras, la mayor parte del coeficiente de valor agregado nacional contenido en las exportaciones corresponde al valor agregado directo: en la industria automovilística y en la de equipos eléctricos asciende al 55\% y en la industria electrónica al $60 \%$. El menor peso que tiene el valor agregado indirecto resulta del débil encadenamiento entre los sectores directamente exportadores y las actividades que producen insumos contenidos en los bienes exportados. De esto se deriva la limitada capacidad de estas exportaciones para generar ingresos en otros sectores de la economía nacional.

xiv. Dada la relevancia que tiene el componente indirecto del valor agregado como indicador de la densidad de las relaciones entre los sectores exportadores y el resto de la economía, en las dos últimas columnas del cuadro 2 se muestra la proporción que en las exportaciones de los sectores representan el valor agregado indirecto de origen manufacturero y el que es creado dentro de las ramas manufactureras integrantes del sector exportador (columna "indirecto intraindustrial"). Como se aprecia, respecto del conjunto de las exportaciones manufactureras, el valor agregado indirecto de origen manufacturero representa el 4,3\% de las exportaciones, proporción que en la IME se reduce a 1,6\%. Esta muestra de debilidad de los encadenamientos internos de las exportaciones manufactureras con el resto de la manufactura también se manifiesta dentro de cada una de las ramas exportadoras, particularmente en la IME. Por ejemplo, en la industria de computación y electrónica, tan relevante en materia de exportaciones, el valor agregado indirecto originado en otras ramas del sector equivale solo a un $0,2 \%$ del valor de las exportaciones del sector. 


\section{VI}

\section{Conclusiones}

El objetivo del presente trabajo ha sido contribuir a explicar el hecho de que no obstante que el sector exportador mexicano ha registrado un dinamismo y una maduración extraordinarios en las últimas décadas, su contribución al crecimiento del conjunto de la economía ha sido débil.

En la perspectiva que aquí se ha adoptado, esto se fundamenta en el hecho de que la expansión de las exportaciones manufactureras está débilmente encadenada con el mercado interno. Esto se explica porque el valor agregado nacional contenido en las exportaciones es relativamente bajo, especialmente en las exportaciones de la industria maquiladora, que aporta más del $60 \%$ de las exportaciones manufactureras del país.

El valor agregado de origen nacional contenido en las exportaciones se divide entre directo e indirecto. En la medida en que los encadenamientos internos respecto del abastecimiento de partes e insumos para las actividades exportadoras son más densos, la proporción de valor agregado indirecto en el total del valor agregado nacional contenido en las exportaciones será más elevada. En el trabajo se ha mostrado que en la manufactura exportadora mexicana prima el valor agregado directo, lo que indica el relativo aislamiento entre los sectores exportadores y el resto de la economía nacional.

\section{ANEXO ESTADÍSTICO}

CUADRO A 1

México: composición de las exportaciones de la industria manufacturera, 2003 (En millones de pesos)

\begin{tabular}{|c|c|c|c|c|c|c|c|c|}
\hline \multirow{2}{*}{ Subsector } & \multicolumn{2}{|c|}{ Total } & \multicolumn{2}{|c|}{ Economía interna (EI) } & \multicolumn{2}{|c|}{$\begin{array}{l}\text { Industria maquiladora } \\
\text { de exportación (IME) }\end{array}$} & \multicolumn{2}{|c|}{ Porcentajes } \\
\hline & Exportaciones & Porcentaje & Exportaciones & Porcentaje & Exportaciones & Porcentaje & EI & IME \\
\hline Industria alimentaria & 24186 & 1,8 & 18873 & 3,7 & 5312 & 0,6 & 78 & 22 \\
\hline Industria de las bebidas y del tabaco & 14795 & 1,1 & 13981 & 2,8 & 814 & 0,1 & 94,5 & 5,5 \\
\hline Fabricación de insumos textiles & 16804 & 1,3 & 6631 & 1,3 & 10174 & 1,2 & 39,5 & 60,5 \\
\hline Confección de productos textiles & 11103 & 0,8 & 2549 & 0,5 & 8554 & 1 & 23 & 77 \\
\hline Fabricación de prendas de vestir & 73418 & 5,5 & 15323 & 3 & 58096 & 7 & 20,9 & 79,1 \\
\hline Fabricación de productos de cuero & 7511 & 0,6 & 2944 & 0,6 & 4567 & 0,6 & 39,2 & 60,8 \\
\hline Industria de la madera & 2363 & 0,2 & 1061 & 0,2 & 1302 & 0,2 & 44,9 & 55,1 \\
\hline Industria del papel & 9240 & 0,7 & 4030 & 0,8 & 5211 & 0,6 & 43,6 & 56,4 \\
\hline Impresión e industrias conexas & 3977 & 0,3 & 1196 & 0,2 & 2781 & 0,3 & 30,1 & 69,9 \\
\hline $\begin{array}{l}\text { Fabricación de productos del petróleo } \\
\text { y del carbón }\end{array}$ & 14794 & 1.1 & 14791 & 29 & 4 & 0 & 100 & 0 \\
\hline Industria química & 46117 & 3,5 & 40792 & 8 & 5325 & 0,6 & 88,5 & 11,5 \\
\hline Industria del plástico y del hule & 37055 & 2,8 & 10100 & 2 & 26954 & 3,3 & 27,3 & 72,7 \\
\hline $\begin{array}{l}\text { Fabricación de productos de } \\
\text { minerales no metálicos }\end{array}$ & 18523 & 1,4 & 11309 & 2,2 & 7214 & 0,9 & 61,1 & 38,9 \\
\hline Industrias metálicas básicas & 34172 & 2,6 & 27346 & 5,4 & 6825 & 0,8 & 80 & 20 \\
\hline Fabricación de productos metálicos & 42803 & 3,2 & 19137 & 3,8 & 23666 & 2,9 & 44,7 & 55,3 \\
\hline Fabricación de maquinaria y equipo & 43406 & 3,3 & 24048 & 4,7 & 19358 & 2,3 & 55,4 & 44,6 \\
\hline Fabricación de equipo electrónico & 385317 & 28,9 & 47741 & 9,4 & 337576 & 40,8 & 12,4 & 87,6 \\
\hline Fabricación de equipo eléctrico & 122366 & 9,2 & 23135 & 4,6 & 99231 & 12 & 18,9 & 81,1 \\
\hline Fabricación de equipo de transporte & 366969 & 27,5 & 211203 & 41,6 & 155766 & 18,8 & 57,6 & 42,4 \\
\hline Fabricación de muebles & 18256 & 1,4 & 4415 & 0,9 & 13841 & 1,7 & 24,2 & 75,8 \\
\hline Otras industrias manufactureras & 41990 & 3,1 & 6488 & 1,3 & 35501 & 4,3 & 15,5 & 84,5 \\
\hline Total exportaciones & 1335165 & 100 & 507093 & 100 & 828072 & 100 & 38 & 62 \\
\hline
\end{tabular}

Fuente: estimación de los autores sobre la base de Instituto de Estadística y Geografía (INEGI), "Matriz de insumo-producto 2003", México, D.F., 2008. 


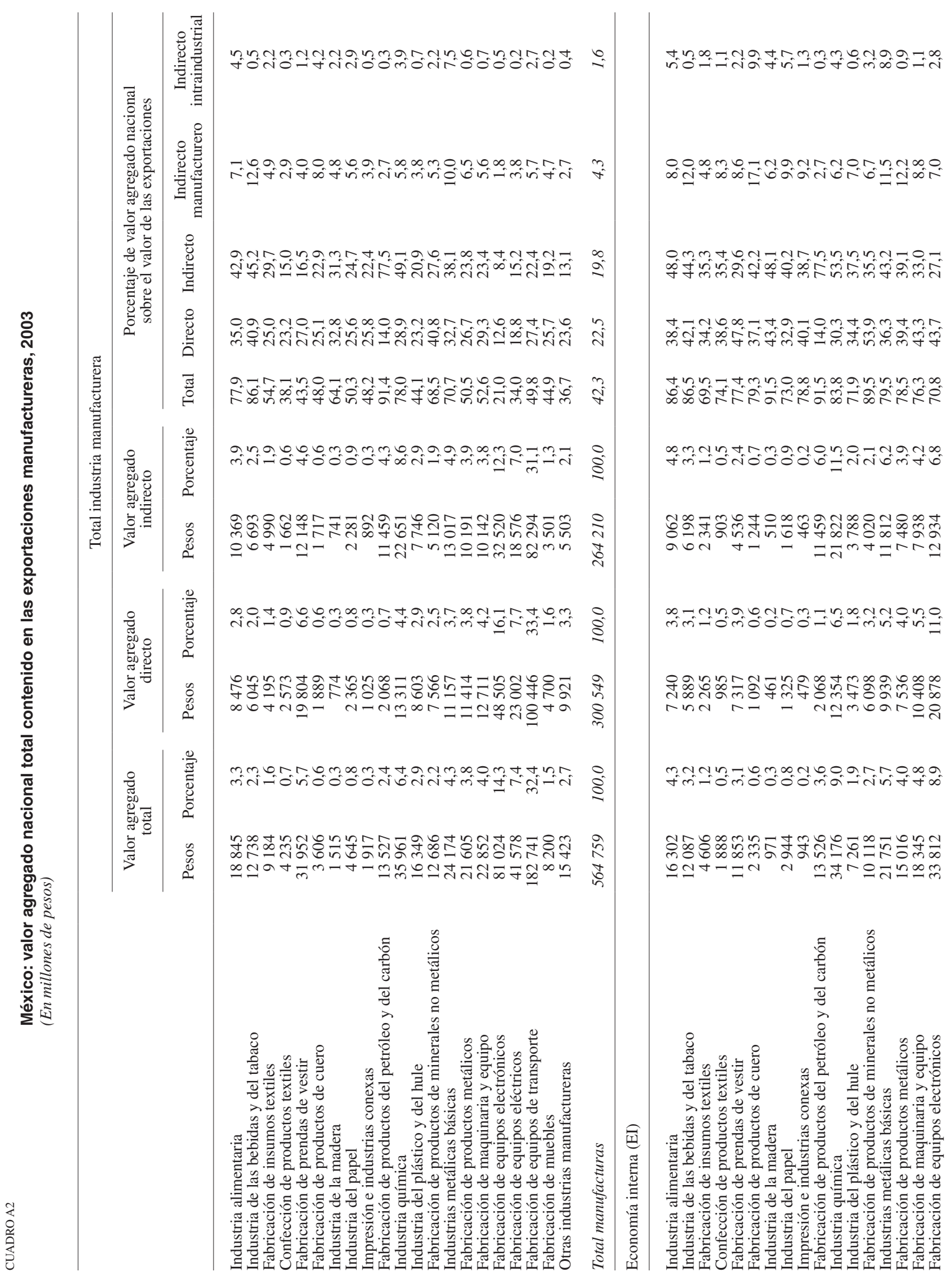




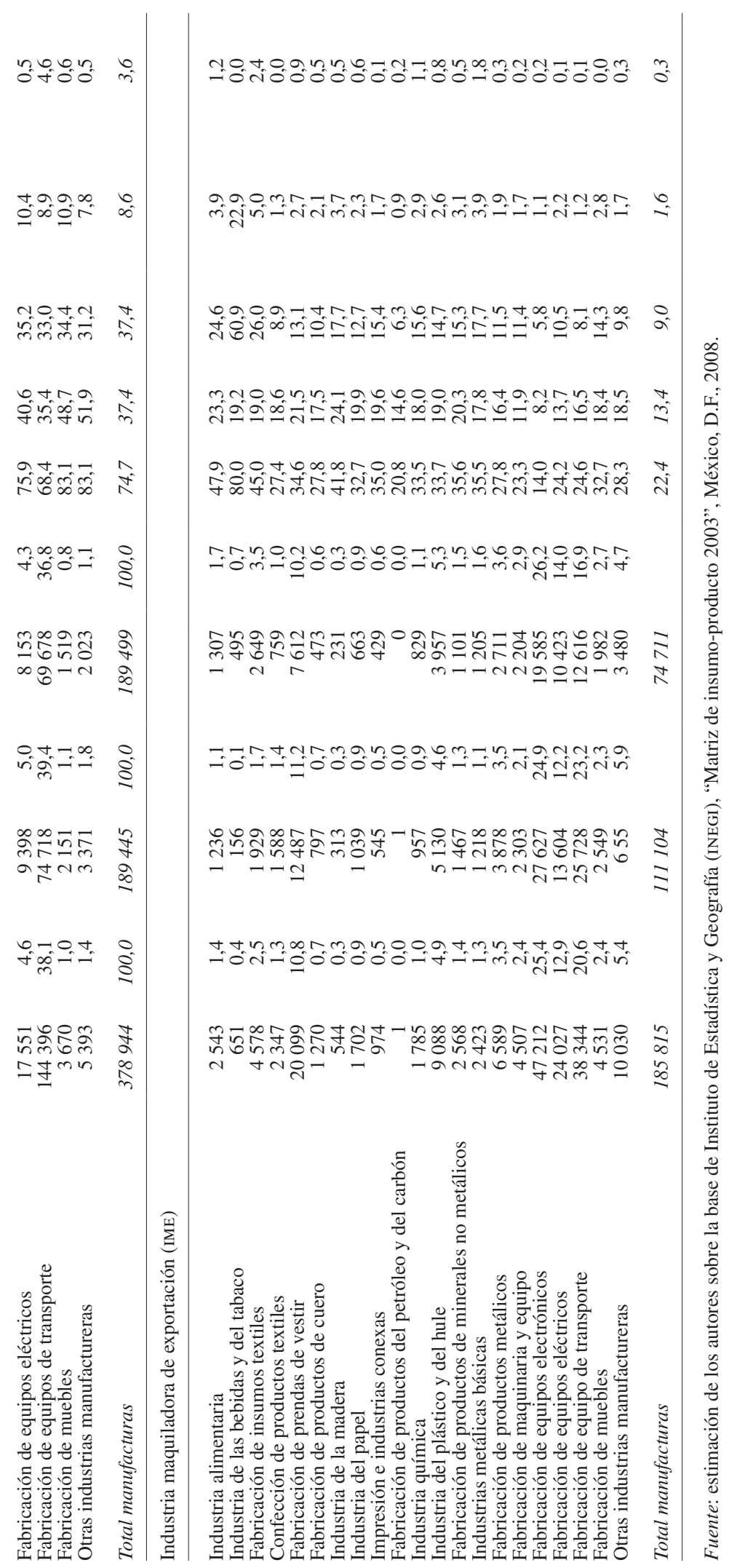




\section{Bibliografía}

Akyüz, Y. (2010), "Export dependence and sustainability of growth in China and the East Asian production network", Research Paper, $\mathrm{N}^{\mathrm{0}} 27$, South Centre, abril.

Banco de México (2009), Informe anual 2008, México, D.F.

Banco Mundial (2011), "World DataBank" [en línea] http://databank. worldbank.org/data/home.aspx

Bhagwati, J. y T. Srinivasan (1979), "Trade policy and development", International Economic Policy: Theory and Evidence, R. Dornbusch y J. Frenkel (eds.), Baltimore, Johns Hopkins University Press.

Breda, E. y R. Cappariello (2008), "A tale of two bazaar economies: an input-output analysis of Germany and Italy", Roma, Banco de Italia.

Breda, E., R. Cappariello y R. Zizza (2007), "Measures of the external trade impulse to economic growth: how relevant is the internationalization of production?", Roma, Banco de Italia.

CEPAL (Comisión Económica para América Latina y el Caribe) (2008), Panorama de la inserción internacional de América Latina y el Caribe 2007. Tendencias 2008 (LC/G.2383-P), Santiago de Chile. Publicación de las Naciones Unidas, $\mathrm{N}^{\circ}$ de venta: S.08.II.G.36.

Cervantes, R. (2008), “Apertura comercial y empleo en México, 19802004", tesis de doctorado, México, D.F., Facultad de Economía, Universidad Nacional Autónoma de México.

Chen, X. y otros (2008), "Domestic value added and employment generated by Chinese exports: a quantitative estimation", MPRA Paper, $\mathrm{N}^{\mathrm{o}} 15663$, Munich, Munich University Library.

(2005), "The estimation of domestic value added and employment induced by exports: an application to Chinese exports to the United States", documento presentado en la reunión de la American Economic Association, Filadelfia.

Daudin, G., C. Rifflart y D. Schweisguth (2009), "Who produces for whom in the world economy", Document de travail, N 2009-18, París, Observatoire Français des Conjonctures Économiques.

Eatwell, J. (1998), "Import substitution and export-led growth", The New Palgrave. A Dictionary of Economics, J. Eatwell, M. Milgate y P. Newman (eds.), vol. 2, Londres, The Macmillan Press.

Feder, G. (1983), "On exports and economic growth", Journal of Development Economics, vol. 12, № 1-2, Amsterdam, Elsevier.

Felipe, J. (2003), "Is export-led growth passe? Implications for developing Asia", Economics Working Papers, № 48, Manila, Banco Asiático de Desarrollo.

Felipe, J. y J. Lim (2005), "Export or domestic-led growth in Asia?", Economics Working Papers, $\mathrm{N}^{\circ}$ 69, Manila, Banco Asiático de Desarrollo.

Grossman, G.M. y E. Helpman (1991), Innovation and Growth in the Global Economy, Cambridge, Massachusetts, MIT Press.

Harrod, R. (1933), International Economics, Nueva York, Harcourt, Brace and Company.

Hausmann, R., J. Hwang y D. Rodrik (2007), "What you export matters", Journal of Economic Growth, vol. 12, № 1, Springer.

He, D. y W. Zhang (2010), "How dependent is the Chinese economy on exports and in what sense has its growth been export-led?", Journal of Asian Economics, vol. 21, № 1, Amsterdam, Elsevier.

Helpman, E. y P. Krugman (1985), Market Structure and Foreign Trade, Cambridge, Massachusetts, MIT Press.
Hicks, J. (1950), A Contribution to the Theory of the Trade Cycle, Oxford, Clarendon Press.

INEGI (Instituto Nacional de Estadística y Geografía) (2008), "Matriz de insumo producto de México 2003" [en línea] http://www.inegi. org.mx/est/contenidos/proyectos/scn/c_anuales/matrizinsumo/ default.aspx

Kaldor, N. (1989), The Essential Kaldor, Nueva York, Holmes \& Meier.

Kohli, I. y N. Singh (1989), "Exports and growth: critical minimum effort and diminishing returns", Journal of Development Economics, vol. 30, No 2, Amsterdam, Elsevier.

Koopman, R., Z. Wang y S.- J. Wei (2008), "How much of Chinese exports is really made in China? Assessing domestic valueadded when processing trade is pervasive", NBER Working Paper, $\mathrm{N}^{\circ} 14109$, Cambridge, Massachusetts, National Bureau of Economic Research.

Kranendonk, H. y J. Verbruggen (2008), "Decomposition of GDP growth in European countries. Different methods tell different stories", CPB Document, $\mathrm{N}^{\circ} 158$, СРB Netherlands Bureau for Economic Policy Analysis, $\mathrm{N}^{\circ} 158$, enero.

Krueger, A. (1980), "Trade policy as an input to development", American Economic Review, vol. 70, № 2, Nashville, Tennessee, American Economic Association.

Loschky, A. y L. Ritter (2006), "Import Content of Exports" (sTD/ NAES/TASs/ITs(2006)) 16, París, Organización de Cooperación y Desarrollo Económicos (OCDE), septiembre.

Moreno-Brid, J.C., J.C. Rivas y J. Santamaría (2005), "Mexico: economic growth, exports and industrial performance after NAFTA", serie Estudios y perspectivas, № 42 (LC/L.2479-P), México, D.F., sede subregional de la CEPAL en México. Publicación de las Naciones Unidas, $\mathrm{N}^{\mathrm{o}}$ de venta: E.06.II.G.6.

Myro Sánchez, R. y otros (2008), Globalización y deslocalización. Importancia y efectos para la industria española, Madrid, Ministerio de Industria, Turismo y Comercio.

Palley, T.I. (2002), "A new development paradigm: domestic demandled growth. Why it is needed \& how to make it happen", Foreign Policy in Focus, Albuquerque, septiembre.

Palma, J.G. (2005), "The seven 'stylized facts' of the Mexican economy since trade liberalization and NAFTA", Industrial and Corporate Change, vol. 14, N ${ }^{\circ}$ 6, Oxford, Oxford University Press.

Razmi, A. y R.A. Blecker (2008), "Developing country exports of manufactures: moving up the ladder to escape the fallacy of composition", Journal of Development Studies, vol. 44, $\mathrm{N}^{\mathrm{o}} 1$, Taylor \& Francis, enero.

Ruiz Nápoles, P. (2004), "Exports, growth, and employment in Mexico, 1978-2000", Journal of Post Keynesian Economics, vol. 27, $N^{\circ} 1$, M.E. Sharpe Inc.

Smith, A. (1958), Investigación de la naturaleza y causas de la riqueza de las naciones, Fondo de Cultura Económica. Publicado originalmente en 1776.

Thirlwall, A.P. (1979), "The balance of payments constraint as an explanation of international growth rate differences", Banca Nazionale del Lavoro Quarterly Review, vol. 32, № 128, Banca Nazionale del Lavoro, marzo. 